\title{
Case series: Three cases of dermatitis artefacta
}

\section{Gangadhar Harsha, Jithin Bijina, Anjan Kumar Patra}

\author{
Department of Dermatology, MVJ Medical College and Research Hospital,Bangalore, India
}

Corresponding author: Dr. Gangadhar Harsha, E-mail: harshagangadhar5@gmail.com

\begin{abstract}
Three cases of dermatitis artefacta are reported for their varied presentation, diagnostic indicators and complex management. A 13 year old young child presented with multiple painful erosions with crusting over face, upper limb, lower limb trunk since 1 month. Histopathology was non specific. A 21 year old man presented with multiple asymptomatic raised lesions on the right forearm since one year. His brother claimed that the individual had initially produced these lesions using burning cigarette ends. A 25 year old medical student presented with multiple reddish lesions over all fingers of his hands and lips. He was depressed, withdrawn, and unwilling to discuss his problems and had interpersonal conflict with his parents. Psychiatric evaluation and follow-up was essential in all cases.
\end{abstract}

Key words: Dermatitis artefacta; Psychotherapy; Psychocutaneous disorders

\section{INTRODUCTION}

Dermatitis artefacta is a factitious disorder leading to deliberately and consciously self inflicted injuries aimed at satisfying unconscious emotional needs; classically, the patient perpetuates the lesions and denies having induced the same. Factitial dermatitis or dermatitis artefacta is a psychocutaneous disorder produced by or perpetuated by the patient's own actions.

\section{CASE REPORT}

\section{Case 1}

A 13 year old young child presented with multiple painful erosions with crusting over face, upper limb, lower limb trunk since 1 month. Patient and mother claimed that lesions were sudden in onset with burning sensation prior to onset of lesions and refused application of irritants, self infliction of injury.

On examination: there were multiple linear, monomorphic erosions with crusting clearly demarcated from surrounding skin over face, upper limb, lower limb, trunk, nape of neck, buttocks mainly over the accessible areas (Figs. la - ld). Psychiatric evaluation was done and revealed teenage maladjustment disorder.
Individual patient counselling and parental counselling was done. Patient was treated with fluoxetine $10 \mathrm{mg}$ and Olanzapine $2.5 \mathrm{mg}$ and topical fusidic acid over erosions.

\section{Case 2}

A 21 year old man presented with multiple asymptomatic raised lesions on the right forearm of one year duration. He gave a history of progressive appearance of lesions on the affected limb. The mode of onset could not be explained.

On examination: there were multiple hyperpigmented atrophic scars in linear distribution on the dorsal aspect of right forearm (Fig. 2). A skin biopsy revealed findings suggestive of hypertrophic scars. He was treated with occlusive/intralesional steroids over a three-month peiod with fare response. His brother claimed that the individual had initially produced these lesions using burning cigarette ends. No motive could be identified for producing these lesions over this length of time. He was placed under regular therapy and observation under the care of a psychiatrist.

\section{Case 3}

A 25 year old medical student presented to us with multiple erosions over all fingers of his hands and also over

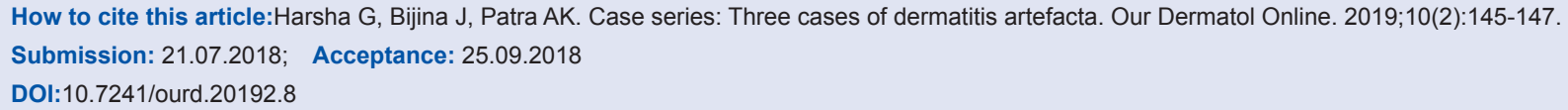




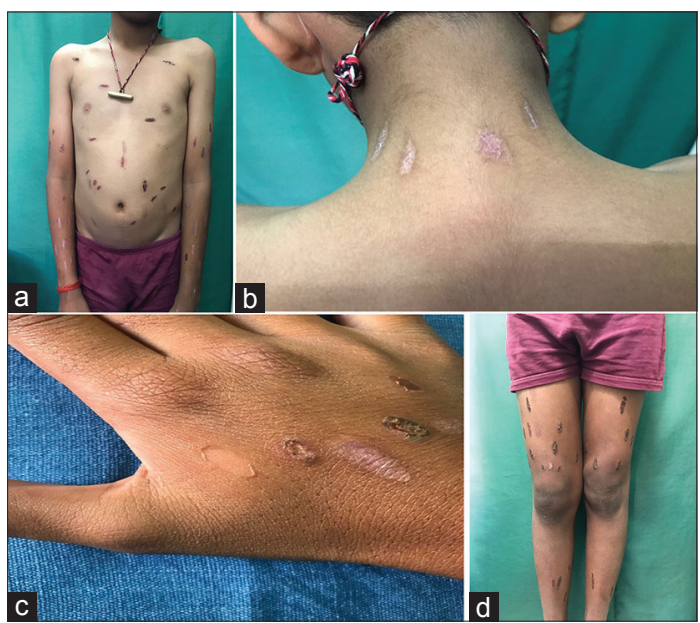

Figure 1: (a) Multiple linear, monomorphic erosions with crusting clearly demarcated from surrounding skin over upper limb, trunk. (b) Multiple healed erosions over nape of neck. (c) Multiple fresh and healed erosions with crust over dorsum of right hand. (d) Multiple linear, monomorphic erosions with crusting clearly demarcated from surrounding skin over lower limb.

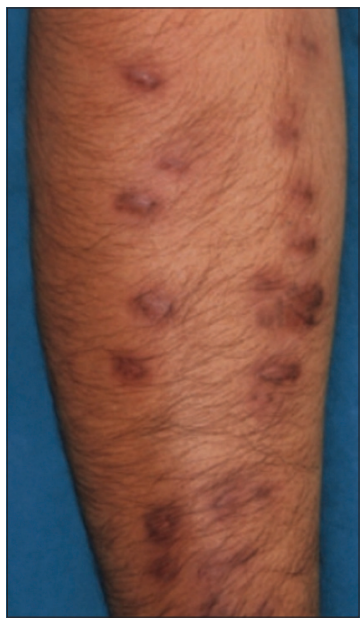

Figure 2: Multiple hyperpigmented atrophic scars in linear distribution on the dorsal aspect of right forearm.

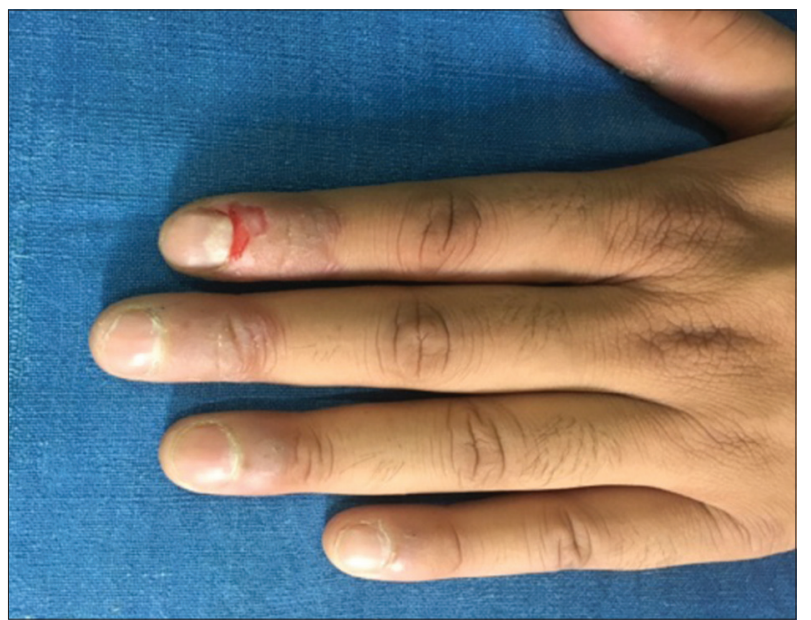

Figure 3: Multiple erosions with post inflammatory hyperpigmentation over tip and proximal nail folds of all fingers. lips. He claims that these lesions appear spontaneously associated with burning sensation. He was depressed, withdrawn, and unwilling to discuss his problems.

On examination: there were multiple erosions with post inflammatory hyperpigmentation over tip and proximal nail folds of all fingers and lips (Fig. 3). On psychiatric evaluation, we came to the conclusion that interpersonal conflict with his parents was the most probable cause. His histopathology was nonspecific. He was treated with antidepressants and psychiatric counselling was done.

\section{DISCUSSION}

Dermatitis artefacta, a factitious dermatitis from self-inflicted injury, is a diagnosis of exclusion and when there is evidence of an artifactual cause. Lack of clinical and histopathologic correlation can raise suspicion, as in this case. Dermatitis artefacta frequently is associated with underlying psychosocial problems $[1,2]$. Therefore, confrontation to explore the underlying psychosocial conflicts should be strongly discouraged [3]; rather, a gentle, nonjudgmental, and empathetic approach often works. Several factors such as delayed developmental milestones, marital dispute, loss of close relatives in the recent past, selfguilt, disturbed parent-child relationship, bipolar personality disorders, and sexual and substance abuse are implicated as the precipitating factors [4].

The management of these patients has to be gentle, non-confrontational and flexible and involves building a mutual trust and rapport between patient and doctor. It is imperative that we follow an integral approach and treat these patients as a bio-psychosocial individual incorporating their thoughts and manipulations without being judgment. Initial strong therapeutic alliance with the patient, in terms of mutual trust and rapport, is very crucial for a better outcome as prognosis of the disease is not good with frequent waxing and waning. Antidepressants in the form of selective serotonin reuptake inhibitor and behavioral therapy [5] are the mainstay of treatment. Dermatological care with bland emollient, topical antibiotics, and occlusive dressing should not be underestimated as the patients tend to be emotionally attached to their skin.

\section{CONCLUSION}

DA is often a challenge for the clinicians because of its rarity, vague history, bizarre and polymorphic 
morphology, lack of decisive diagnostic tests, and poor therapeutic outcomes.

\section{CONSENT}

The examination of the patient was conducted according to the Declaration of Helsinki principles.

\section{REFERENCES}

1. Mohandas P, Bewley A, Taylor R. Dermatitis artefacta and artefactual skin disease: the need for a psychodermatology multidisciplinary team to treat a difficult condition. Br J Dermatol. 2013;169:600-6.

2. Gil-Bistes D, Kluger N, Guillot B, Bessis D. Dermatitis artefacta in a young girl. Arch Pediatr. 2010;17:1543-5.

3. Gattu S, Rashid RM, Khachemoune A. Self-induced skin lesions: A review of dermatitis artefacta. Cutis. 2009;84:247-51.

4. Zalewska A, Kondras K, Narbutt J, Sysa-Jedrzejowska A. Dermatitis artefacta in a patient with paranoid syndrome. Acta Dermatovenerol Alp Pannonica Adriat. 2007;16:37-9.

5. Heller MM, Koo JM. Neurotic excoriations, acne excoriee, and factitial dermatitis. In: Heller MM, Koo JY, editors. Contemporary Diagnosis and Management in Psychodermatology. $1^{\text {st }}$ ed. Newton, PA, USA: Handbooks in Health Care Co; 2011. pp. 37-44.

Copyright by Gangadhar Harsha, et al. This is an open-access article distributed under the terms of the Creative Commons Attribution License, which permits unrestricted use, distribution, and reproduction in any medium, provided the original author and source are credited.

Source of Support: Nil, Conflict of Interest: None declared. 